\title{
POWER OSCILLATION DAMPING CONTROL STRATEGIES FOR FACTS DEVICES USING LOCALLY MEASURABLE QUANTITIES
}

\author{
James F. Gronquist William A. Sethares Fernando L. Alvarado Robert H. Lasseter \\ University of Wisconsin-Madison, Madison, Wisconsin, USA
}

\begin{abstract}
This paper presents a way to derive power oscillation damping control strategies for Flexible AC Transmission (FACTS) devices, and derives these laws for the four major types of FACTS devices using an energy function (Lyapunov) method. All controls rely only on locally measurable information, and are independent of system topology, implying structural uncertainty need not affect power oscillation damping control strategies. Keywords: FACTS, power oscillation damping, energy function methods
\end{abstract}

\section{Introduction}

FACTS devices are increasingly being used as cost effective measures to increase power system transmission capability, to improve first swing margin, to actively damp oscillations, and to help stabilize weakly coupled systems in the event of critical faults [5], [6]. This paper presents a new method for designing power swing damping control strategies for FACTS devices, and derives these laws for thyristor controlled series capacitors (TCSC), static VAR compensators (SVC), static condensers (STATCON), and thyristor controlled phase shifting transformers (TCPS). Previous approaches have either assumed a particular control strategy (and then demonstrated the stability of the system [3]), or have chosen an a priori set of variables to estimate (and then to control based upon inferred values of these variables [4]).

The present approach introduces a structure preserving energy function in which each FACTS device in the system is identified with a specific term. Taking the derivative of this energy function, it is easy to see how to choose parameters within the FACTS devices to ensure that each term of the derivative is negative semidefinite, and hence that each term contributes to system stability. This strategy identifies both the control variables, and control laws as a function of these variables. Important aspects of the approach are:

1. All oscillation damping controls rely on strictly locally measurable information.

95 Wh 185-9 PWRS A paper recommended and approved by the IEEE Power System Engineering Committee of the IEEE Power Engineering Society for presentation at the 1995 IEEE/PES Winter Meeting, January 29, to Februàry 2, 1995, New York, NY. Manuscript submitted August 1, 1994; made available for printing November $23,1994$.
2. The control laws are independent of the system topology.

3. No linearization is performed in deriving these control laws. It is therefore expected they will have a larger region of validity than controls designed based upon linearized system models.

The second statement implies that for any system configuration (provided it meets certain stability criteria), the same control law can be used for a specific device type no matter where it is located. This also implies that the control law does not require detailed knowledge of the configuration of the system. Thus structural uncertainties in the network do not impact the form of the control law, at least as far as power swing damping control is concerned.

For TCSC, SVC, and STATCON, given a system and a compensator location scheme, system stability can be formally established. For TCPS, numerical results show the effectiveness of a proposed control law, but without a formal proof of stability. Section 2 presents the system model. Section 3 presents the energy function (Lyapunov candidate) and control laws, and Section 4 presents simulations showing the effectiveness of the derived control laws. More detailed derivations are included in the appendices.

\section{System Model}

The system is assumed to have $N_{m}$ generators, each modeled as a constant voltage behind a transient reactance. The generators supply power to a system of $N_{b}$ buses, interconnected via lossless transmission lines.

Define the quantities:

- $P_{k}^{m}$ is the mechanical input power for generator $k$. It is assumed constant.

- $E_{k}, \delta_{k}, \omega_{k}$ is the voltage, rotor angle, and deviation frequency for generator $k$

- $b_{k k}$ is the transient susceptance for generator $k$

- $H_{k}$ is the inertia constant for generator $k$

- $v_{k}, \theta_{k}$ are voltage magnitude and angle for bus $k$.

- $P_{k}$, and $Q_{k}\left(v_{k}\right)$ are the real and reactive load at bus $k . P_{k}$ is assumed to be constant, $Q_{k}\left(v_{k}\right)$ may vary as a function of bus voltage magnitude.

- $b_{k m}$ is the susceptance of the $(k, m)$ branch 
- $\alpha_{k m}, \beta_{k m}$, are the tap ratio magnitude, and tap phase shift angle for any transformer in the $(k, m)$ branch

Since the system is lossless, assume that the power injected into the system is equal to the power removed, that is, $\sum_{k=1}^{N_{m}} P_{k}^{m}=\sum_{k=1}^{N_{b}} P_{k}$. Assume also that bus $k$ is connected to generator $k$ by admittance $-j b_{k k}$ for $1 \leq$ $k \leq N_{m}$. Thus buses 1 to $N_{m}$ are generator terminal buses.

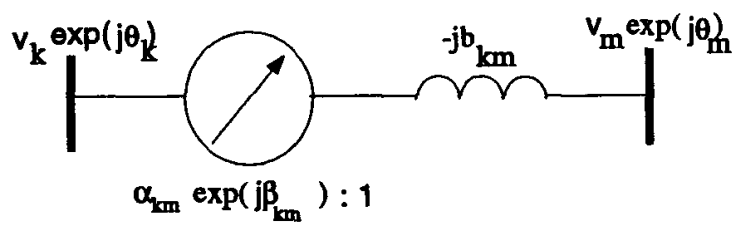

Fig. 1: Transformer Model

Transformers are modeled using the nodal admittance matrix:

$$
\left[\begin{array}{c}
I_{k} \\
I_{m}
\end{array}\right]=\left(-j b_{k m}\right)\left[\begin{array}{cc}
\frac{1}{\left|t_{k m}\right|^{2}} & -\frac{1}{t_{k m}^{*}} \\
-\frac{1}{t_{k m}} & 1
\end{array}\right]\left[\begin{array}{c}
V_{k} \\
V_{m}
\end{array}\right]
$$

where $t_{k m}$ is the complex tap ratio, $t_{k m}=\alpha_{k m} e^{j \beta_{k m}}$, $I_{k}$ and $I_{m}$ are the complex injected currents into nodes $k$ and $m, V_{k}$ and $V_{m}$ are the complex voltages at nodes $k$ and $m, t_{k m}^{*}$ is the complex conjugate of $t_{k m}$, and $k$ is the tap side. TCPS will be modeled as a transformer which injects a voltage $r_{k m} v_{k}$ in quadrature, where $r_{k m}$ is the control parameter. For this case, $t_{k m}=\frac{1}{1+j r_{k m}}$, $\alpha_{k m}=\frac{1}{\sqrt{1+r_{k m}^{2}}}$, and $\beta_{k m}=-\tan ^{-1}\left(r_{k m}\right)$. Let $\hat{r}_{k m}$ be the steady state set point of the TCPS in the $(k, m)$ branch, and $\tilde{r}_{k m}$ be the damping control modulation.

SVC and STATCON are modeled as variable reactive loads by considering $Q_{k}\left(v_{k}\right)=\hat{Q}_{k}\left(v_{k}\right)+\tilde{Q}_{k}\left(v_{k}\right)$. $\tilde{Q}_{k}\left(v_{k}\right)$ is the compensator control modulation, and $\hat{Q}_{k}\left(v_{k}\right)$ takes into account any uncontrolled reactive load and the steady state set point of any compensator at bus $k$.

TCSC are modeled as variable series line admittances $b_{k m}=\hat{b}_{k m}+\tilde{b}_{k m}$, where $\tilde{b}_{k m}$ is the damping control modulation for the TCSC at the $(k, m)$ branch and where $\hat{b}_{k m}$ is the line admittance of the $(k, m)$ branch plus the admittance of the steady state set point of any TCSC in the $(k, m)$ branch.

The dynamics for each generator are described by :

$$
\begin{aligned}
\dot{\delta}_{k} & =\omega_{k} \\
H_{k} \dot{\omega}_{k} & =P_{k}^{m}-b_{k k} E_{k} v_{k} \sin \left(\delta_{k}-\theta_{k}\right) \quad\left(=F_{k}\right)
\end{aligned}
$$

Additionally, algebraic constraints at each bus are obtained by summing real power injections and reactive current injections

$$
\begin{aligned}
f_{k} & =-P_{k}+b_{k k} E_{k} v_{k} \sin \left(\delta_{k}-\theta_{k}\right)+\sum_{m=1, m \neq k}^{N_{m}} P_{k m}=0 \\
g_{k} & =-\frac{Q_{k}\left(v_{k}\right)}{v_{k}}+b_{k k}\left(E_{k} \cos \left(\delta_{k}-\theta_{k}\right)-v_{k}\right)
\end{aligned}
$$

$$
+\sum_{m=1, m \neq k}^{N_{b}} \frac{Q_{k m}}{v_{k}}=0
$$

where $P_{k m}$ is the real power injected into bus $k$ by the $(k, m)$ branch, and $\frac{Q_{k m}}{v_{k}}$ is the reactive current injected into bus $k$ by the $(k, m)$ branch. Appendix A gives expressions for $P_{k m}$ and $\frac{Q_{k m}}{v_{k}}$.

In the absence of active swing damping modulation, the above equations represent the entire set of differential/algebraic equations (DAE) governing the evolution of the power system. For this case, $\delta_{k}$ and $\omega_{k}$ are the dynamic states, while $v_{k}$ and $\theta_{k}$ are dependent variables whose values are uniquely determined via the $f_{k}$ and $g_{k}$ equations, (provided that the implicit function theorem can be applied, i.e. that the system is not operating at the 'Point of Collapse'). It will be shown later that the proposed feedback laws exploit the structure of the system, in effect creating 'new' dynamic states.

\section{Energy Function and Control}

Given the above system model, it is possible to split the $f_{k}$ and $g_{k}$ into two components $f_{k}=\hat{f}_{k}+\tilde{f}_{k}$, and $g_{k}=\hat{g}_{k}+\tilde{g}_{k}$, where $\hat{f}_{k}$ and $\hat{g}_{k}$ contain no contributions due to the swing damping modulation parameters $\tilde{Q}_{k}\left(v_{k}\right), \tilde{b}_{k m}, \tilde{r}_{k m}$. Thus all action of the control law is captured in the $\tilde{f}$ and $\tilde{g}$ terms. See Appendix B for how this is accomplished.

A Lyapunov candidate function $\mathcal{V}$ is defined much as in [2], [3], [7]. A formal definition is provided in Appendix C. Without active control, the state trajectory will always follow the contours of constant $\mathcal{V}$ since the system is assumed lossless. When the active control is implemented, the energy of the system decreases as long as the $\tilde{f}$ and $\tilde{g}$ terms are chosen in a sensible way.

Somewhat more formally, $\mathcal{V}$ is a valid Lyapunov function provided:

1. The equilibrium of the system is a critical point of $\mathcal{V}$, that is, the gradient of $\mathcal{V}$ with respect to a minimal state representation is zero at equilibrium. ${ }^{1}$

2. The Hessian of $\mathcal{V}$ with respect to this minimal state representation evaluated at the equilibrium is positive definite.

3. The derivative of $\mathcal{V}$ with respect to time is negative semi-definite.

For the case of no active control modulation, it is a straightforward exercise to evaluate the Hessian. For the case of active control, 'new' dynamic states are created via the control feedback. The Hessian matrix should reflect derivatives with respect to these 'new' states. See Appendix D for how this new Hessian matrix is evaluated.

In the absence of active damping control modulation, it is straight-forward to identify a minimal state representation, but for the case where active control modulation is implemented via feedback of measured system quantities, this identification is not always trivial.

\footnotetext{
${ }^{1}$ A minimal state representation is one which has the fewest possible number of state variables.
} 
Differentiating $\mathcal{V}$ with respect to time yields an expression containing terms in $\hat{f}, \hat{g}, \tilde{f}$ and $\tilde{g}$. The hat terms are not affected by the control signals, while the tilde terms can be used to ensure that the derivative is always decreasing. This is carried out in detail in Appendix C.

The objective a stable control is to drive $\mathcal{V}$ to its minima quickly, and this can be accomplished by a suitable choice of a control law for each FACTS device.

- For TCSC, choose $\tilde{b}_{k m}=\Gamma_{k m}\left(V_{k m} \dot{V}_{k m}\right)$, where $\Gamma_{k m}(x)>0$ for $x>0, \Gamma_{k m}(x)<0$ for $x<0$, and $\Gamma_{k m}(0)=0$, and where $V_{k m}$ is the magnitude of the voltage drop across the $(k, m)$ branch. Assuming that an accurate model of the transmission line into which the TCSC is inserted is available, then $V_{k m}$ is locally measurable. Specifically, if the line has impedance $X_{k m}$ then $V_{k m}=X_{k m} I_{k m}+V_{c}$ where $V_{c}$ is the magnitude of the voltage drop across the TCSC. $\dot{V}_{k m}$ can be inferred based upon tracking of $V_{k m}$.

- For SVC or STATCON, choose $\tilde{Q}_{k}=\Lambda_{k}\left(\frac{\dot{v}_{k}}{v_{k}}\right)$, where $\Lambda_{k}(x)>0$ for $x>0, \Lambda_{k}(x)<0$ for $x<0$, and $\Lambda_{k}(0)=0$, and where $v_{k}$ is the terminal voltage magnitude of the compensator at bus $k$. Clearly, this is locally measurable.

- For TCPS, use $\tilde{r}_{k m}=\Upsilon_{k m}\left(\kappa_{k m}, \lambda_{k m}\right)$, $\kappa_{k m}=b_{k m}\left(\frac{d}{d t}\left(v_{k} v_{m} \sin \left(\theta_{m}-\theta_{k}\right)\right)-\hat{r}_{k m} \frac{d}{d t}\left(v_{k}^{2}\right)\right)$, and $\lambda_{k m}=-\frac{1}{2} b_{k m} \frac{d}{d t}\left(v_{k}^{2}\right) . \Upsilon_{k m}(x, y)$ is chosen so that $x \Upsilon_{k m}(x, y)+y \Upsilon_{k m}^{2}(x, y) \leq 0$ for all $(x, y)$, and $\Upsilon_{k m}(0,0)=0$. An example of such a function is $\Upsilon_{k m}(x, y)=-x$ for $y<1$ and $\Upsilon_{k m}(x, y)=$ $-x \exp (1-y)$ for $y>1$. Given the transformer model, we can measure tap and impedance side voltage magnitudes, and currents, and therefore infer the above quantities. The control law for TCPS can be chosen in many ways.

There is a wide range of possible choices for the control functions $\Gamma_{k m}$, and $\Lambda_{k}$. Figure 2 shows a few of the possibilities. Any function which is zero at $x=0$ and whose graph is restricted to the first and third quadrants of the plane is suitable. Some choices will of course be better than others.

Intuitively, these control laws make sense. The TCSC control law operates by opposing changes in the line voltage. For instance, if the magnitude of the voltage drop across the line increases, the TCSC increases line admittance (decreases line impedance), thereby more tightly 'coupling' the ends of the line together. Conversely, if the magnitude of the voltage drop across the line decreases, the TCSC opposes this change by decreasing line admittance. The SVC/STATCON control law has a similar interpretation. If a compensator's terminal voltage is dropping, it should act to oppose that change via capacitive reactive power injection. If the terminal voltage is rising, this change should be opposed via inductive reactive power injection.

For TCPS, if we can assume angular dynamics dominate the voltage dynamics during the transient, the ' $x$ '

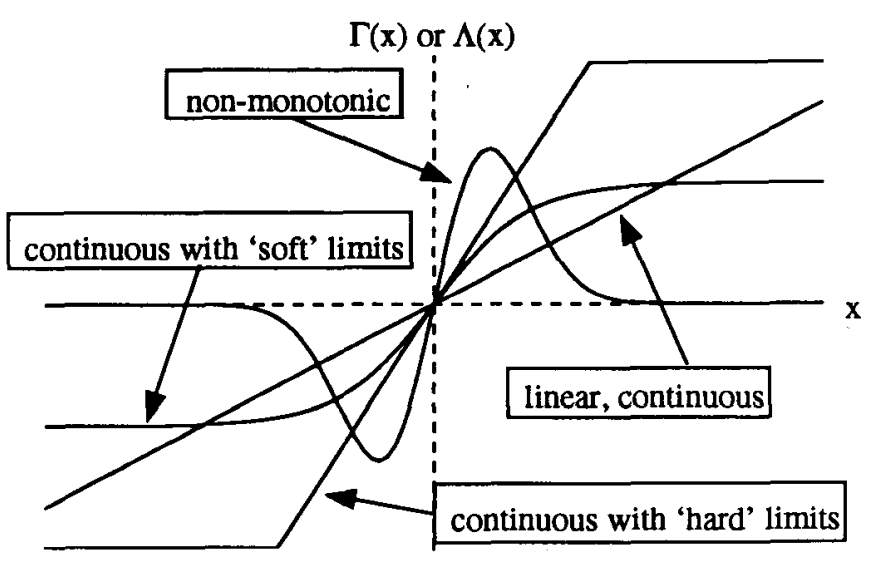

Fig. 2: Control Function Examples. For $\mathrm{\Gamma}, x$ means $V_{k m} \dot{V}_{k m}$, for $\Lambda, x$ means $\dot{v}_{k} / v_{k}$.

term dominates the ' $y$ ', and $x \approx b_{k m} v_{k} v_{m} \frac{d}{d t} \sin \left(\theta_{m}-\right.$ $\theta_{k}$ ). If the angular difference $\theta_{m}-\theta_{k}$ is increasing, the TCPS 'pulls' against this by choosing $\tilde{r}_{k m} \leq 0$, which tends to drive $\frac{d}{d t}\left(\theta_{m}-\theta_{k}\right)$ to zero, or in others words, drive $\theta_{m}-\theta_{k}$ to a constant value.

If machine damping is included the Lyapunov candidate is not affected, while its derivative gains a negative quadratic term in $\boldsymbol{\omega}$. Therefore, the Lyapunov derivative is negative semi-definite in the absence of control, and the control serves to increase this damping.

\section{Simulations}

The system used for the simulations is that of [5], shown in Figure 3. All simulations show a fault being applied to the EF, circuit \#1 line, with the fault being cleared at $t=0.2$ seconds by tripping this line. Machine damping is not included in these simulations, therefore all damping is due strictly to the action of the various FACTS devices. Figure 4 shows the machine responses in the absence of control.

Figure 5 shows the effect of two SVCs in the system, one at the mid-point of line $\mathrm{AE}$, the other at bus $\mathrm{F}$, each rated at \pm 200 MVAR. Though oscillations are being damped, the damping is slow.

Figure 6 shows the effect of two TCSCs in the system, one in the DG line, and the other in the AE line, each rated for 50 percent maximum compensation. Here damping is better than that for SVC.

Figure 7 shows the system response when two TCPS are placed in the system, one in the AE line with the tap at $E$, the other in the DG line with the tap at G. Here, extremely good damping is apparent. Transformer range is plus or minus 0.1 p.u. quadrature voltage injection.

\section{Conclusion}

This paper has presented power oscillation damping control strategies for FACTS devices based upon locally measurable variables, and which are independent 


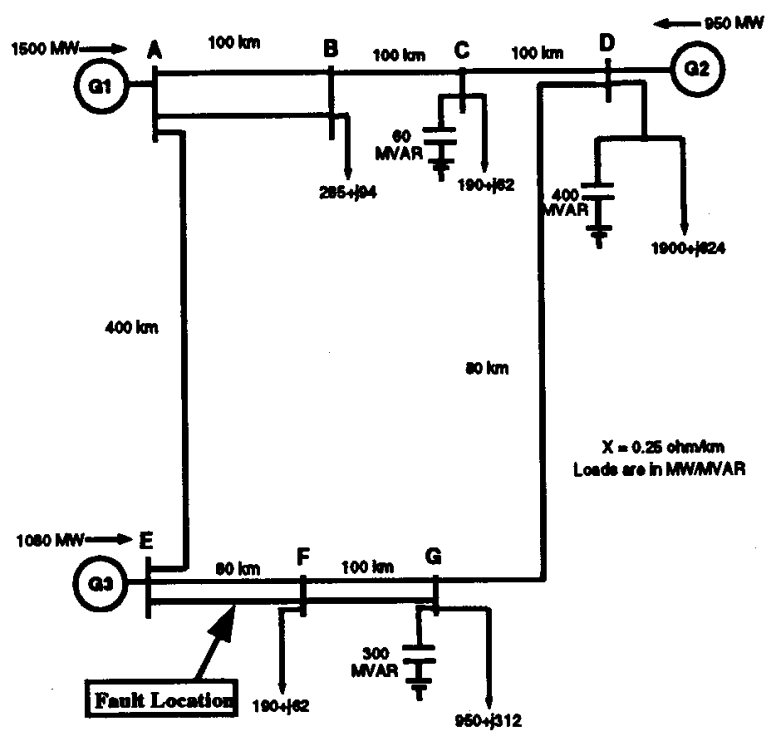

Fig. 3: Test System from [5]

of system topology. The simulations have shown the effectiveness of the control strategies for a variety of compensator placements. This work shows that structural uncertainties in the power system need not impact power oscillation control strategy. Additionally, the authors believe this represents the first time phase shifting transformers have been successfully incorporated into energy function methods.

\section{Acknowledgements}

This research is funded under NSF grant ECS9216520 and EPRI grant RP8030-02. The authors would also like to gratefully acknowledge the contributions of C. DeMarco and I. Dobson for general technical support during the course of this research.

\section{REFERENCES}

[1] T. Athay, R. Podmore, S. Virmani, "A Practical Method for the Direct Analysis of Transient Stability," IEEE Transactions on Power Apparatus and Systems, Vol. PAS-98, No. 2, Mar/Apr 1979, pp. 573-584

[2] A. Bergen and D. Hill, "A Structure Preserving Model for Power System Stabjlity Analysis," IEEE Transactions on Power Apparatus and Systems, Vol. PAS-100, No. 1, Jan. 1981, pp. 25-35

[3] I. Hiskens and D. Hill, "Incorporation of SVCs into Energy Function Methods," IEEE Transactions on Power Systems, Vol. 7, No. 1, Feb. 1992, pp. 133-140

[4] E. Lerch, D. Povh and L. Xu, "Advanced SVC Control for Damping Power System Oscillations," IEEE Transactions on Power Systems, Vol. 6, No. 2, May 1991

[5] D. Povh, "Advantages of Power Electronic Equipment in AC Systems," International Colloquium on HVDC and FACTs Systems, Wellington, New Zealand, 29 Sept. 1993

[6] D. Povh and R. Mihalic, "Enhancement of Transient Stability on AC Transmission by means of Controlled

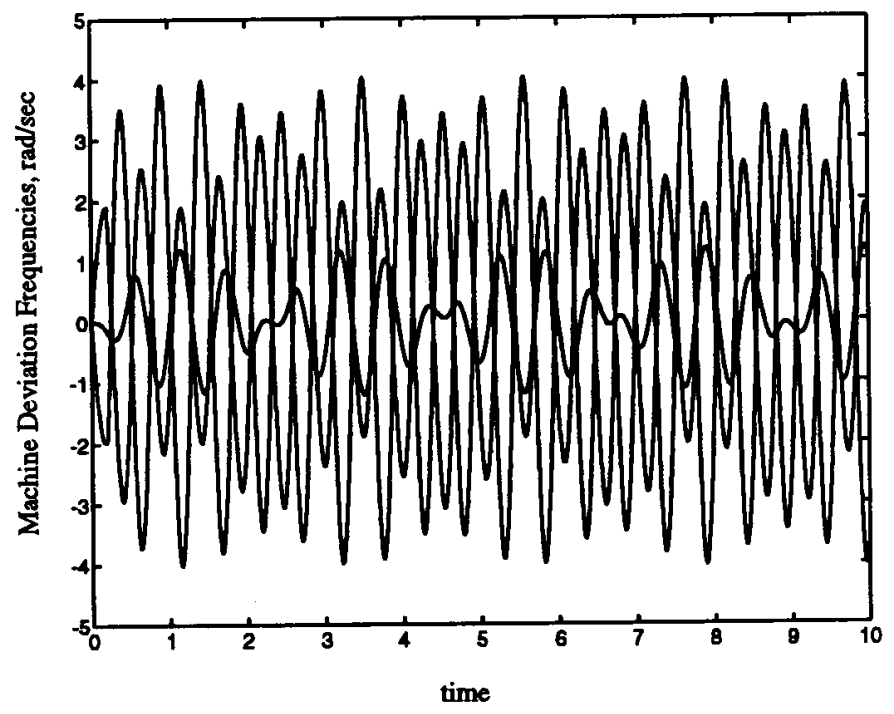

Fig. 4: Uncompensated system deviation frequencies, $\omega$, for a $200 \mathrm{msec}$. three-phase fault at the indicated location.

Series and Parallel compensation," 5th International Conference on $A C$ and $D C$ Power Transmission, 17-20 Sept. 1991, London

[7] N. Tsolas, A. Arapostathis, and P. Varaiya, "A Structure Preserving Energy Function for Power System Transient Stability Analysis," IEEE Transactions on Circuits and Systems, Vol. CAS-32, No. 10, Oct. 1985, pp. 1041-1049

\section{Appendix A: Bus Injection Equations}

If there is no transformer in the $(k, m)$ branch then

$$
\begin{aligned}
P_{k m} & =b_{k m} v_{k} v_{m} \sin \left(\theta_{m}-\theta_{k}\right) \\
\frac{Q_{k m}}{v_{k}} & =b_{k m}\left(v_{m} \cos \left(\theta_{m}-\theta_{k}\right)-v_{k}\right)
\end{aligned}
$$

If bus $k$ is the tap side of a transformer branch $(k, m)$ then

$$
\begin{aligned}
P_{k m} & =b_{k m} \frac{v_{k} v_{m}}{\alpha_{k m}} \sin \left(\theta_{m}-\theta_{k}+\beta_{k m}\right) \\
\frac{Q_{k m}}{v_{k}} & =\frac{b_{k m}}{\alpha_{k m}}\left(v_{m} \cos \left(\theta_{m}-\theta_{k}+\beta_{k m}\right)-\frac{v_{k}}{\alpha_{k m}}\right)
\end{aligned}
$$

If bus $k$ is the impedance side of a transformer branch $(k, m)$ then

$$
\begin{aligned}
P_{k m} & =b_{k m} \frac{v_{k} v_{m}}{\alpha_{k m}} \sin \left(\theta_{m}-\theta_{k}-\beta_{k m}\right) \\
\frac{Q_{k m}}{v_{k}} & =b_{k m}\left(\frac{v_{m}}{\alpha_{k m}} \cos \left(\theta_{m}-\theta_{k}-\beta_{k m}\right)-v_{k}\right)
\end{aligned}
$$

If bus $k$ is the tap side of a TCPS branch $(k, m)$ then

$$
\begin{aligned}
P_{k m}= & b_{k m} v_{k} v_{m}\left[\sin \left(\theta_{m}-\theta_{k}\right)-r_{k m} \cos \left(\theta_{m}-\theta_{k}\right)\right] \\
\frac{Q_{k m}}{v_{k}}= & b_{k m}\left[v_{m}\left(\cos \left(\theta_{m}-\theta_{k}\right)+r_{k m} \sin \left(\theta_{m}-\theta_{k}\right)\right)-\right. \\
& \left.v_{k}\left(1+r_{k m}^{2}\right)\right]
\end{aligned}
$$




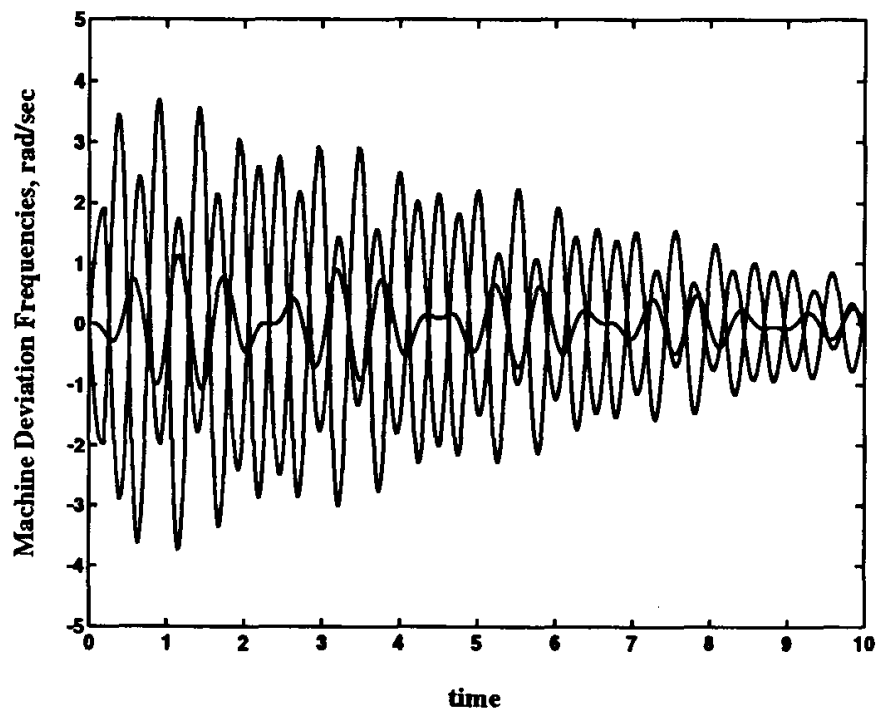

Fig. 5: Simulation with two \pm 200 MVAR SVC, one at the mid-point of line $A E$, the other at bus $F$.

If bus $k$ is the impedance side of a TCPS branch $(k, m)$ then

$\begin{aligned} P_{k m} & =b_{k m} v_{k} v_{m}\left[\sin \left(\theta_{m}-\theta_{k}\right)+r_{k m} \cos \left(\theta_{m}-\theta_{k}\right)\right] \\ \frac{Q_{k m}}{v_{k}} & =b_{k m}\left[v_{m}\left(\cos \left(\theta_{m}-\theta_{k}\right)-r_{k m} \sin \left(\theta_{m}-\theta_{k}\right)\right)-v_{k}\right]\end{aligned}$

Appendix B: Load Flow Equation Partitioning

$$
\begin{aligned}
f_{k} & =\hat{f}_{k}+\tilde{f}_{k} \quad(=0) \\
\hat{f}_{k} & =-P_{k}+b_{k k} E_{k} v_{k} \sin \left(\delta_{k}-\theta_{k}\right)+\sum_{m=1, m \neq k}^{N_{b}} \hat{P}_{k m} \\
\tilde{f}_{k} & =\sum_{m=1, m \neq k}^{N_{b}} \tilde{P}_{k m} \\
g_{k} & =\hat{g}_{k}+\tilde{g}_{k}(=0) \\
\hat{g}_{k} & =-\frac{\hat{Q}_{k}}{v_{k}}+b_{k k}\left(E_{k} \cos \left(\delta_{k}-\theta_{k}\right)-v_{k}\right)+\sum_{m=1, m \neq k}^{N_{b}} \frac{\hat{Q}_{k m}}{v_{k}} \\
\tilde{g}_{k} & =-\frac{\tilde{Q}_{k}}{v_{k}}+\sum_{m=1, m \neq k}^{N_{b}} \frac{\tilde{Q}_{k m}}{v_{k}}
\end{aligned}
$$

where:

- If the $(k, m)$ branch does not have a TCSC installed, or if it is a non-TCPS transformer branch, then $\hat{P}_{k m}=P_{k m}, \frac{\dot{Q}_{k m}}{v_{k}}=\frac{Q_{k m}}{v_{k}}$ defined as in Appendix $A$ and $\tilde{P}_{k m}=\frac{\bar{Q}_{k m}}{v_{k}}=0$

- If the $(k, m)$ branch is has a TCSC installed then

$$
\begin{aligned}
& \hat{P}_{k m}=\hat{b}_{k m} v_{k} v_{m} \sin \left(\theta_{m}-\theta_{k}\right) \\
& \tilde{P}_{k m}=\tilde{b}_{k m} v_{k} v_{m} \sin \left(\theta_{m}-\theta_{k}\right)
\end{aligned}
$$

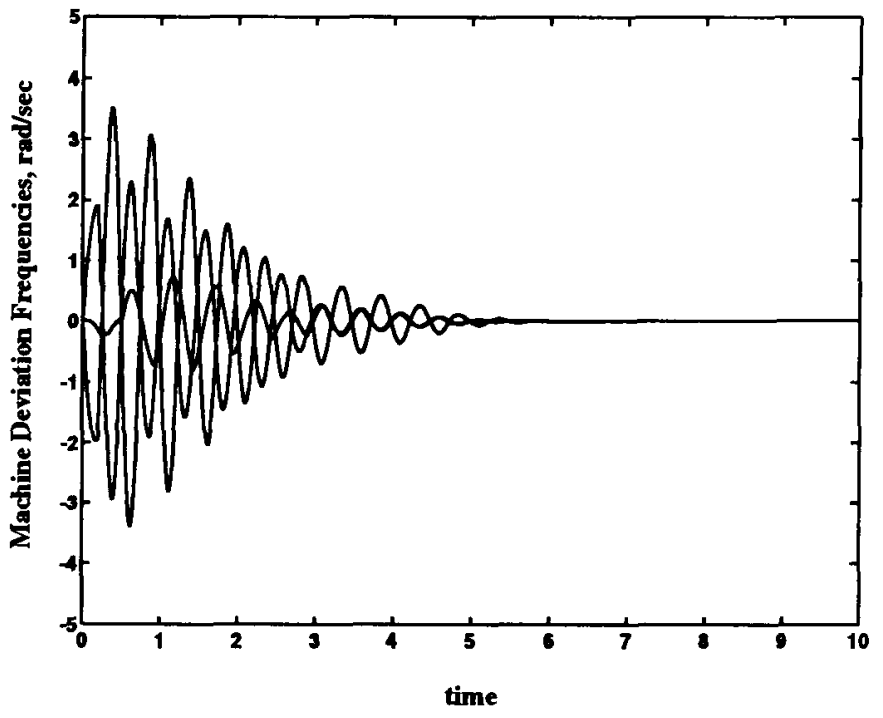

Fig. 6: Simulation with two TCSC placed in the system, one in line $D G$, the other in line $A E$, each rated for $50 \%$ maximum compensation.

$$
\begin{aligned}
& \frac{\hat{Q}_{k m}}{v_{k}}=\hat{b}_{k m}\left(v_{m} \cos \left(\theta_{m}-\theta_{k}\right)-v_{k}\right) \\
& \frac{\tilde{Q}_{k m}}{v_{k}}=\tilde{b}_{k m}\left(v_{m} \cos \left(\theta_{m}-\theta_{k}\right)-v_{k}\right)
\end{aligned}
$$

- If the $(k, m)$ branch has a TCPS installed, and $k$ is the tap side, then

$$
\begin{aligned}
\hat{P}_{k m}= & b_{k m} v_{k} v_{m}\left[\sin \left(\theta_{m}-\theta_{k}\right)-\hat{r}_{k m} \cos \left(\theta_{m}-\theta_{k}\right)\right] \\
\tilde{P}_{k m}= & -b_{k m} v_{k} v_{m} \tilde{r}_{k m} \cos \left(\theta_{m}-\theta_{k}\right) \\
\frac{\hat{Q}_{k m}}{v_{k}}= & b_{k m}\left[v_{m}\left(\cos \left(\theta_{m}-\theta_{k}\right)+\hat{r}_{k m} \sin \left(\theta_{m}-\theta_{k}\right)\right)\right. \\
& \left.-v_{k}\left(1+\hat{r}_{k m}^{2}\right)\right] \\
\frac{\tilde{Q}_{k m}}{v_{k}}= & b_{k m}\left[v_{m} \tilde{r}_{k m} \sin \left(\theta_{m}-\theta_{k}\right)-\right. \\
& \left.v_{k}\left(2 \hat{r}_{k m} \tilde{r}_{k m}+\tilde{r}_{k m}^{2}\right)\right]
\end{aligned}
$$

- If the $(k, m)$ branch has a TCPS installed, and $k$ is the impedance side, then

$$
\begin{aligned}
\hat{P}_{k m}= & b_{k m} v_{k} v_{m}\left[\sin \left(\theta_{m}-\theta_{k}\right)+\hat{r}_{k m} \cos \left(\theta_{m}-\theta_{k}\right)\right] \\
\tilde{P}_{k m}= & b_{k m} v_{k} v_{m} \tilde{r}_{k m} \cos \left(\theta_{m}-\theta_{k}\right) \\
\frac{\hat{Q}_{k m}}{v_{k}}= & b_{k m}\left[v_{m}\left(\cos \left(\theta_{m}-\theta_{k}\right)-\hat{r}_{k m} \sin \left(\theta_{m}-\theta_{k}\right)\right)\right. \\
& \left.-v_{k}\right] \\
\frac{\tilde{Q}_{k m}}{v_{k}}= & -b_{k m} v_{m} \tilde{r}_{k m} \sin \left(\theta_{m}-\theta_{k}\right)
\end{aligned}
$$

\section{Appendix C: Lyapunov Candidate and Control} Law Derivations

This appendix defines the Lyapunov function and derives control laws for $\tilde{Q}_{k}\left(v_{k}\right), \tilde{b}_{k m}$, and $\tilde{r}_{k m}$ which 


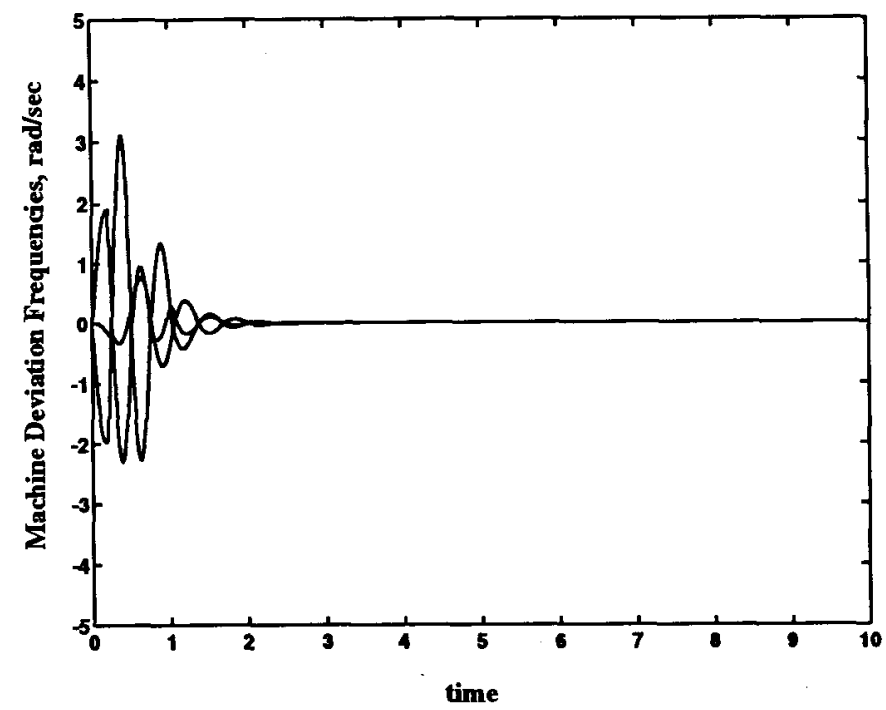

Fig. 7: Simulation with two TCPS placed in the system, one in line $A E$ with tap at $E$, the other in line $D G$ with tap at $G$.

cause the derivative to become negative semi-definite. The system has four types of branches: uncompensated, fixed transformer, TPSC, and TCSC branches. Each branch type contributes a different term to the Lyapunov Candidate. The branches are partitioned by defining the sets:

- $A_{k}=\left\{m, k<m \leq N_{b}\right.$ such that: no. TCSC, TCPS, or transformer is in the $(k, m)$ branch $\}$

- $B_{k}=\left\{m, k<m \leq N_{b}\right.$ such that there is a TCSC in the $(k, m)$ branch $\}$

- $C_{k}=\left\{m \leq N_{b}\right.$ such that a transformer is in the $(k, m)$ branch, and $k$ is the tap side $\}$

- $T_{k}=\left\{m \leq N_{b}\right.$ such that a TCPS is in the $(k, m)$ branch, and $k$ is tap side $\}$

Then, for the $(k, m)$ branch, its $(k, m)$ pair belongs to one and only one of $\bigcup_{k=1}^{N_{b}} A_{k}, \bigcup_{k=1}^{N_{b}} B_{k}, \bigcup_{k=1}^{N_{b}} C_{k}$ or $\bigcup_{k=1}^{N_{b}} T_{k}$.

A Lyapunov candidate function can be defined as:

$$
\begin{aligned}
\mathcal{V}= & \sum_{k=1}^{N_{m}}\left[\frac{1}{2} H_{k} \omega_{k}^{2}-P_{k}^{m} \delta_{k}+\frac{1}{2} b_{k k} V_{k k}^{2}\right] \\
& +\sum_{k=1}^{N_{b}}\left[P_{k} \theta_{k}+\int \frac{\hat{\Omega}_{k}\left(v_{k}\right)}{v_{k}} d v_{k}\right] \\
& +\sum_{k=1}^{N_{b}} \sum_{m \in A_{k}} \frac{1}{2} b_{k m} V_{k m}^{2} \\
& +\sum_{k=1}^{N_{b}} \sum_{m \in B_{k}} \frac{1}{2} \hat{b}_{k m} V_{k m}^{2} \\
& +\sum_{k}^{N_{b}} \sum_{m \in C_{k}} \frac{1}{2} b_{k m} E_{k m}^{2} \\
& +\sum_{k=1}^{N_{b}} \sum_{m \in T_{k}} \frac{1}{2} b_{k m} \hat{F}_{k m}^{2}
\end{aligned}
$$

where:

$$
\begin{gathered}
V_{k k}^{2}=E_{k}^{2}+v_{k}^{2}-2 E_{k} v_{k} \cos \left(\delta_{k}-\theta_{k}\right) \\
V_{k m}^{2}=v_{k}^{2}+v_{m}^{2}-2 v_{k} v_{m} \cos \left(\theta_{m}-\theta_{k}\right)
\end{gathered}
$$

$$
\begin{gathered}
E_{k m}^{2}=\left(\frac{v_{k}}{\alpha_{k m}}\right)^{2}+v_{m}^{2}-2\left(\frac{v_{k}}{\alpha_{k m}}\right) v_{m} \cos \left(\theta_{m}-\theta_{k}+\beta_{k m}\right) \\
\hat{F}_{k m}^{2}=\begin{array}{c}
v_{k}^{2}\left(1+\hat{r}_{k m}^{2}\right)+v_{m}^{2}-2 v_{k} v_{m}\left(\cos \left(\theta_{m}-\theta_{k}\right)\right. \\
\left.+\hat{r}_{k m} \sin \left(\theta_{m}-\theta_{k}\right)\right)
\end{array}
\end{gathered}
$$

Observe that $\frac{\partial \mathcal{V}}{\partial \delta_{k}}=-F_{k}, \frac{\partial \mathcal{V}}{\partial v_{k}}=-\hat{g}_{k}, \frac{\partial \mathcal{V}}{\partial \theta_{k}}=-\hat{f}_{k}$, and $\frac{\partial \mathcal{V}}{\partial \omega_{k}}=H_{k} \omega_{k}$.

Differentiating $\mathcal{V}$ with respect to time yields :

$$
\frac{d \mathcal{V}}{d t}=\sum_{k=1}^{N_{b}}\left[\tilde{f}_{k} \dot{\theta}_{k}+\tilde{g}_{k} \dot{v}_{k}\right]
$$

In the absence of active damping control, i.e. $\tilde{Q}_{k}\left(v_{k}\right)=\tilde{b}_{k m}=\tilde{r}_{k m}=0 \quad \forall k, m, \frac{d \mathcal{V}}{d t} \equiv 0$, the state trajectory follows contours of constant $\mathcal{V}$.

Further manipulation of equation 5 yields:

$$
\left.\begin{array}{rl}
\frac{d \mathcal{V}}{d t}= & -\sum_{k=1}^{N_{b}} \frac{\tilde{Q}_{k}\left(v_{k}\right)}{v_{k}} \dot{v}_{k} \\
& +\sum_{k=1}^{N_{b}-1} \sum_{m=k+1}^{N_{b}} \\
& +\sum_{k=1}^{N_{b}-1} \sum_{m=k+1}^{N_{b}}\left[\frac{\tilde{Q}_{k m}}{v_{k}} \dot{v}_{k}+\frac{\tilde{Q}_{m k}}{v_{m}} \dot{v}_{m}\right] \\
\tilde{P}_{k m} \dot{\theta}_{k}+\tilde{P}_{m k} \dot{\theta}_{m}
\end{array}\right]
$$

The first summation above can be interpreted as the sum over all shunt compensated buses of the contribution of the compensator at that bus. The second and third summations taken together can be interpreted as the contribution of all compensated branches (TCSC or TCPS). Yet further manipulation equation 6 yields:

$$
\begin{aligned}
\frac{d \mathcal{V}}{d t}= & -\sum_{k=1}^{N_{b}} \frac{\tilde{Q}_{k}\left(v_{k}\right)}{v_{k}} \dot{v}_{k} \\
& -\sum_{k=1}^{N_{b}} \sum_{m \in B_{k}} \tilde{b}_{k m} V_{k m} \dot{V}_{k m} \\
& +\sum_{k=1}^{N_{b}} \sum_{m \in T_{k}} u_{k m}
\end{aligned}
$$

where $\mathcal{U}_{k m}$ is defined as:

$$
\begin{aligned}
\frac{\mathcal{U}_{k m}}{b_{k m}}= & \tilde{r}_{k m} \frac{d}{d t}\left(v_{k} v_{m} \sin \left(\theta_{m}-\theta_{k}\right)\right) \\
& -\frac{1}{2}\left(2 \hat{r}_{k m} \tilde{r}_{k m}+\tilde{r}_{k m}^{2}\right) \frac{d}{d t}\left(v_{k}^{2}\right) \\
= & \frac{\kappa_{k m} \tilde{r}_{k m}+\lambda_{k m} \tilde{r}_{k m}^{2}}{b_{k m}}
\end{aligned}
$$

The objective is to drive $\mathcal{V}$ to its minima, implying choices of :

- $\tilde{Q}_{k m}\left(v_{k}\right)=\Gamma_{k}\left(\frac{\dot{v}_{k}}{v_{k}}\right)$, where $\Gamma_{k}(x)>0$ for $x>0$, $\Gamma_{k}(x)<0$ for $x<0$, and $\Gamma_{k}(0)=0$

- $\tilde{b}_{k m}=\Lambda_{k m}\left(V_{k m} \dot{V}_{k m}\right)$, where $\Lambda_{k m}(x)>0$ for $x>$ $0, \Lambda_{k m}(x)<0$ for $x<0$, and $\Lambda_{k m}(0)=0$

- $\tilde{r}_{k m}=\Upsilon\left(\kappa_{k m}, \lambda_{k m}\right)$, where $x \Upsilon(x, y)+y \Upsilon^{2}(x, y) \leq 0 \quad \forall x, y$, and $\Upsilon(0,0)=0$

The above choices for $\tilde{Q}_{k m}\left(v_{k}\right), \tilde{b}_{k m}$, and $\tilde{r}_{k m}$ ensure $\frac{d \mathcal{V}}{d t} \leq 0$, implying Lyapunov stability. 


\section{Appendix D: Lyapunov Candidate Spatial Derivatives}

Suppose there are $N_{1}$ shunt compensated buses and $N_{2}$ TCSC compensated lines.

It is assurned throughout this section that the shunt compensator terminal voltage magnitudes, and TCSC branch voltage magnitudes are all independent of one another, that is, none of these quantities can be written as a function of the others. To see how this might fail, consider the case where two SVC's are connected to the same bus - only one of the SVC terminal voltage magnitudes is independent, not both simultaneously.

This section utilizes center-of-angle (COA) coordinates as in [1] where the COA is defined as:

$\delta_{0}=\frac{\sum_{k=1}^{N_{m}} H_{k} \delta_{k}}{\sum_{k=1}^{N_{m}} H_{k}}$

and the center-of-angle velocity is $\omega_{0}=\dot{\delta}_{0}$. The new coordinate angles are $\delta_{k}=\delta_{k}-\delta_{0}$, and $\bar{\theta}_{k}=\theta_{k}-\delta_{0}$. The new machine velocity coordinates become $\bar{\omega}_{k}=\omega_{k}-\omega_{0}$.

Substitution of these new coordinates back into the differential/algebraic equations do not change their form. Substitution into the Lyapunov candidate, $\mathcal{V}$, changes the form only by adding a constant off-set. Therefore, we drop the 'bar' notation and assume all angles and angular velocities are specified in COA.

In COA, it is shown in [1] that

$$
\sum_{k=1}^{N_{m}} H_{k} \delta_{k}=0
$$

which implies

$$
\sum_{k=1}^{N_{m}} H_{k} \omega_{k}=0
$$

Therefore, we can write

$$
\delta_{N_{m}}=-\left(\sum_{k=1}^{N_{m}-1} H_{k} \delta_{k}\right) / H_{N_{m}}
$$

and

$$
\omega_{N_{m}}=-\left(\sum_{k=1}^{N_{m}-1} H_{k} \omega_{k}\right) / H_{N_{m}}
$$

Define

$$
\begin{gathered}
H=\operatorname{diag}\left(H_{1}, H_{2}, \cdots H_{N_{m}-1}\right) \\
a=-\left(H_{1}, H_{2}, \cdots H_{N_{m}-1}\right)^{t} / H_{N_{m}} \\
\delta=\left(\delta_{1}, \delta_{2}, \cdots \delta_{N_{m}-1}\right)^{t} \\
\omega=\left(\omega_{1}, \omega_{2}, \cdots \omega_{N_{m}-1}\right)^{t}
\end{gathered}
$$

Then

$$
\delta_{N_{m}}=a^{t} \delta
$$

and

$$
\omega_{N_{m}}=a^{t} \omega
$$

Also define

$$
F=\left(F_{1}, F_{2}, \cdots F_{N_{m}-1}\right)
$$

If the functional forms of $\tilde{Q}_{k}$ and $\tilde{b}_{k m}$ are substituted into the $f$ and $g$ expressions of appendix B, they take the form:

$$
\begin{gathered}
f\left(\delta, \delta_{N_{m}}, v, \theta,\left[\dot{V}_{k m}\right],\left[V_{k m}\right]\right)=0 \\
g\left(\delta, \delta_{N_{m}}, v, \dot{v}, \theta,\left[\dot{V}_{k m}\right],\left[V_{k m}\right]\right)=0
\end{gathered}
$$

where $\left[V_{k m}\right]$ is the vector of TCSC compensated branch voltage magnitudes.

To simply notation, redefine $\left[V_{k m}\right]=\mu$, where $\mu_{j}$ can be identified with a specific $(k, m)$ pair and there is a TCSC in the $(k, m)$ branch, $\mu \in \mathbf{R}^{N_{2}}$. For each $\mu_{j}$ we can define an additional constraint equation :

$$
M_{j}=v_{k}^{2}+v_{m}^{2}-2 v_{k} v_{m} \cos \left(\theta_{m}-\theta_{k}\right)-\mu_{j}^{2}=0
$$

Partition the $v$ vector into two vectors $v_{a}$ and $v_{b}$, where $v_{a}$ contains all those bus voltages associated with un-compensated buses, and $v_{b}$ contains all those bus voltages associated with compensated buses. Then $v_{a} \in$ $\mathbf{R}^{N_{b}-N_{1}}$ and $v_{b} \in \mathbf{R}^{N_{1}}$. Using the same partition as that for $v$, we can separate $g$ into $g_{a}$ and $g_{b}$.

\section{Implicit Differential Algebraic Set}

Using the definitions above, the complete set of equations governing the power system can be rewritten:

$$
\mathcal{Z}(x, y)=\left[\begin{array}{c}
\dot{\delta}-\omega \\
H \dot{\omega}-F\left(\delta, v_{a}, v_{b}, \theta\right) \\
g_{b}\left(\delta, \delta_{N_{m}}, v_{a}, v_{b}, \dot{v}_{b}, \theta, \mu, \dot{\mu}\right) \\
g_{a}\left(\delta, \delta_{N_{m}}, v_{a}, v_{b}, \theta, \mu, \dot{\mu}\right) \\
f\left(\delta, \delta_{N_{m}}, v_{a}, v_{b}, \theta, \mu, \dot{\mu}\right) \\
M\left(v_{a}, v_{b}, \theta, \mu\right) \\
\delta_{N_{m}}-a^{t} \delta \\
\omega_{N_{m}}-a^{t} \omega
\end{array}\right]=0
$$

where the state vector $x$ is defined as:

$$
x=\left[\omega^{t}, \delta^{t}, v_{b}^{t}, \mu^{t}\right]^{t}
$$

and the $y$ vector denotes those quantities dependent upon $x$

$$
y=\left[\dot{\omega}^{t}, \dot{\delta}^{t}, \dot{v}_{b}^{t}, \dot{\mu}^{t}, v_{a}^{t}, \theta^{t}, \delta_{N_{m}}, \omega_{N_{m}}\right]^{t}
$$

This system is well defined in some open region of the equilibrium, $\left(x^{*}, y^{*}\right)$, provided that $\frac{\partial \mathcal{Z}}{\partial y}$ is non-singular when evaluated at $\left(x^{*}, y^{*}\right)$.

\section{Lyapunov Candidate Spatial Derivatives}

The Lyapunov candidate can be written as:

$$
\mathcal{V}=T\left(\omega, \omega_{N_{m}}\right)+U\left(\delta, \delta_{N_{m}}, v_{a}, v_{b}, \theta\right)
$$

Using $x$ as defined above, the gradient of $\mathcal{V}$ is:

$$
\nabla_{x} \mathcal{V}=\left[\begin{array}{c}
H \omega+a H_{N_{m}} \omega_{N_{m}} \\
-\left[\begin{array}{c}
F \\
\hat{g}_{b} \\
0
\end{array}\right]-\bar{y}_{\bar{x}}^{t}\left[\begin{array}{c}
F_{N_{m}} \\
\hat{g}_{a} \\
\hat{f}
\end{array}\right]
\end{array}\right]
$$


where

$$
\bar{y}_{\bar{x}}=\left[\begin{array}{ccc}
a^{t} & 0 & 0 \\
\frac{\partial v_{a}}{\partial \delta} & \frac{\partial v_{a}}{\partial v_{b}} & \frac{\partial v_{a}}{\partial \mu} \\
\frac{\partial \theta}{\partial \delta} & \frac{\partial \theta}{\partial v_{b}} & \frac{\partial \theta}{\partial \mu}
\end{array}\right]
$$

At the equilibrium $\nabla_{x} \mathcal{V}=0$, since $F=F_{N_{m}}=\omega=$ $\omega_{N_{m}}=\hat{g}_{a}=\hat{g}_{b}=\hat{f}=0$ at equilibrium.

The $\bar{y}_{\bar{x}}$ term is a spatial derivative of variables implicitly dependent upon $x$, with respect to $x$ terms. These can be calculated via $y_{x}=-\mathcal{Z}_{y}^{-1} \mathcal{Z}_{x}$, and extracting those terms of $y_{x}$ which define $\bar{y}_{\bar{x}}$.

The Hessian of $\mathcal{V}$ can be computed as:

$$
\nabla_{x}^{2} \mathcal{V}=\left[\begin{array}{cc}
H+a a^{t} H_{N_{m}} & 0 \\
0 & \mathcal{A}+\mathcal{B}+\mathcal{C}+\mathcal{D}
\end{array}\right]
$$

where:

$$
\begin{gathered}
\mathcal{A}=-\left[\begin{array}{ccc}
\frac{\partial F}{\partial \delta} & \frac{\partial F}{\partial v_{b}} & 0 \\
\frac{\partial \hat{g}_{b}}{\partial \delta} & \frac{\partial \hat{g}_{b}}{\partial v_{b}} & 0 \\
0 & 0 & 0
\end{array}\right] \\
\mathcal{B}=-\left[\begin{array}{ccc}
0 & \frac{\partial F}{\partial v_{a}} & \frac{\partial F}{\partial \theta} \\
\frac{\partial \hat{g}_{b}}{\partial \delta_{N_{m}}} & \frac{\partial \hat{g}_{b}}{\partial v_{a}} & \frac{\partial \hat{g}_{b}}{\partial \theta} \\
0 & 0 & 0
\end{array}\right] \bar{y}_{\bar{x}} \\
\mathcal{C}=-\bar{y}_{\bar{x}}^{t}\left[\begin{array}{ccc}
0 & \frac{\partial F_{N_{m}}}{\partial v_{b}} & 0 \\
\frac{\partial \hat{g}_{a}}{\partial \delta} & \frac{\partial \hat{g}_{a}}{\partial v_{b}} & 0 \\
\frac{\partial \hat{f}}{\partial \delta} & \frac{\partial f}{\partial v_{b}} & 0
\end{array}\right] \\
\mathcal{D}=-\bar{y}_{\bar{x}}^{t}\left[\begin{array}{ccc}
\frac{\partial F_{N_{m}}}{\partial \delta_{N_{m}}} & \frac{\partial F_{N_{m}}}{\partial v_{a}} & \frac{\partial F_{N_{m}}}{\partial \theta} \\
\frac{\partial \hat{g}_{a}}{\partial \delta_{N_{m}}} & \frac{\partial \tilde{g}_{a}}{\partial v_{b}} & \frac{\partial \hat{g}_{a}}{\partial \theta} \\
\frac{\partial f}{\partial \delta_{N_{m}}} & \frac{\partial \hat{f}}{\partial v_{a}} & \frac{\partial \hat{f}}{\partial \theta}
\end{array}\right] \bar{y}_{\bar{x}}
\end{gathered}
$$

